Reprod. Nutr. Dévelop., 1988, 28 Suppl. n 1, 149-150

\title{
Comparaison de différentes méthodes de calcul du temps de séjour des résidus alimentaires dans l'ensemble du tube digestif chez la vache laitière
}

Muriel MAMBRINI, J. L. PEYRAUD, H. RULQUIN

Station de Recherches sur la Vache Laitière, I.N.R.A., Saint-Gilles, 35590 L'Hermitage, France.

Summary. Two compartments time independant model (2C) and one compartment time dependant model (1C) of degree 2 for concentrates and 3 for forages provided estimates of total mean retention time (MRT) in the digestive tract which were similar to direct calculations but more variable. Model $1 \mathrm{C}$ was found superior in fitting the data.

Les modèles utilisés pour décrire le transit des particules marquées à différents niveaux du tube digestif doivent d'abord conduire à une estimation correcte du temps de séjour total (TSMT). Nous avons comparé, chez la vache laitière, la mesure directe de TSMT aux estimations obtenues par ajustement des cinétiques d'excrétion fécale des marqueurs à un modèle à 2 compartiments ou à 1 modèle à 1 compartiment à taux de sortie dépendant du temps.

Matériel et méthodes. Les TSMT du fourrage ( $F$ ) et du concentré (C) ont été mesurés pour 3 rations mixtes à base d'ensilage de maïs $(F / C=65 / 35)$, d'ensilage d'herbe $(F / C=55 / 45)$ et de foin $(F / C=55 / 45)$, distribuées en 2 repas par jour $(8 \mathrm{~h}, 17 \mathrm{~h}$ ) à 2 niveaux d'apport (ad libitum $=18 \mathrm{~kg} \mathrm{MS}$ ingérée $/ \mathrm{j}$ et $80 \%$ de l'ad libitum), respectivement à 4,5 et 6 vaches pie noires produisant $25 \mathrm{~kg}$ de lait $4 \%$. Les fourrages et concentrés, marqués respectivement avec de l'Ytterbium et de l'Europium (Peyraud et al., 1988) ont été distribués simultanément à $8 \mathrm{~h}$ puis les transits de $\mathrm{F}$ et $\mathrm{C}$ ont été suivis par collecte totale des fèces pendant 8 jours.

Les TSMT ont été calculés par la moyenne des temps écoulés entre le repas marqué et le milieu de chaque intervalle de collecte, pondérée par les quantités (méthode S1, Faichney, 1975) ou les concentrations (méthode S2, Thielemans et al., 1978) de marqueur excrété. Ils ont par ailleurs été estimés par ajustement des données d'excrétion fécale au modèle de Grovum et Williams (1973, méthode GW) et à une loi gamma de degré 2 ou 3 (méthode G2 ou G3, Ellis et al., 1979). La méthode $\mathrm{S} 1$ a été prise comme référence car elle ne nécessite pas d'hypothèse sur le flux de MS fécale. Les TSMT obtenus avec les autres méthodes ont été reliés à $\mathrm{S} 1$ selon le modèle : TSMT $=\mathrm{a} \times \mathrm{TSMT} \mathrm{S1}+\mathrm{b}( \pm$ effet « aliment $(\mathrm{F} / \mathrm{C}) \|)$. L'effet des facteurs de variation de TSMT a été testé par analyse de variance (modèle : TSMT = moyenne + effets « ration, aliment, vache, niveau d'apports " + «interactions» + « résiduelle »).

Résultats et discussion. La méthode $\mathrm{S} 2$ a conduit à des résultats identiques à S1 (tabl. 1) ; les irrégularités du flux de MS fécale entre 2 intervalles de collecte ne sont donc pas perceptibles pour le calcul de TSMT. La méthode GW a donné 
en moyenne des estimations de TSMT proches de celles calculées par S1 mais, pour chaque aliment ( $F$ ou $C$ ), GW a tendance à sous-estimer légèrement les TSMT faibles et à surestimer les TSMT les plus élevés (tabl. 1). La méthode G2

TABL. 1. - Estimation du temps de séjour moyen (TSMT) des résidus alimentaires dans l'ensemb/e du tube digestif selon différentes méthodes de calcul.

\begin{tabular}{|c|c|c|c|c|c|c|}
\hline & S1 & $\mathrm{S} 2$ & GW & $\mathrm{G} 2$ & G3 & $\mathrm{G} 2+3$ \\
\hline TSMT fourrages $(h)$ & 55,9 & 56,3 & 56,3 & 62,7 & 54,8 & 54,8 \\
\hline TSMT concentrés (h) & 39.2 & 39,3 & 39,4 & 38,1 & 33,9 & 38,1 \\
\hline Régression avec S1 : écart-type résiduel & - & 0,82 & 2,21 & 3,93 & 2,84 & 3,46 \\
\hline : pente & - & 0,98 & $1,10^{*}$ & 0,98 & 0,94 & 0.94 \\
\hline : ordonnée à l'origine & - & 1,41 & $-4,58^{*}$ & 3,54 & $-0,31$ & 2,00 \\
\hline Analyse de variance $\$$ : écart-type résiduel & 4,5 & 4,3 & 5,2 & 6,9 & 5,5 & $6, ?$ \\
\hline
\end{tabular}

S1 : Faichney (1975); S2 : Thielmans et al. (1978); GW : modèle de Grovum et Williams (1973) ; G2 : loi gamma de degré 2 ; G3 de degré 3 (Ellis et al., 1979); G2 + 3 : association de G2 pour les concentrés et de G3 pour les fourrages: " pente significativement différente de 1 ou ordonnée à l'origine significativement différente de $0(P<0,05)$; $\$$ de modèle: TSMT $=$ moyenne + effets (aliment, ration, animaux, niveau d'apport) + interactions + résiduelle.

a surestimé le TSMT de F de 7 h et $\mathrm{G} 3$ a sous-estimé de 5 h celui de $C$. Pour éviter les biais dans les comparaisons entre aliments, nous avons donc utilisé $\mathrm{G} 2$ pour le transit de $\mathrm{C}$ et $\mathrm{G} 3$ pour celui de $F$ (méthode $\mathrm{G} 2+3$ ).

L'utilisation des modèles a toujours accru la variabilité de la mesure de TSMT par rapport aux mesures directes S1 et S2. Ainsi, l'écart-type résiduel de l'analyse de variance a été identique pour S1 et S2 (4,4 h en movenne). Avec la méthode S1 la variabilité observée entre animaux, toujours très importante (écart-type de $4,5 \mathrm{~h})$, a été proche de celle observée entre régimes $(4,0 \mathrm{~h})$ et supérieure à celle observée entre niveaux d'apport $(1,0 \mathrm{~h})$.

Outre l'estimation de TSMT, le choix d'un modèle repose également sur la qualité de l'ajustement des cinétiques d'excrétion observées. L'écart-type résiduel moyen des ajustements a été plus élevé avec le modèle GW qu'avec G2 et surtout G3 (respectivement 56, 48 et $32 \mathrm{~g}$ de marqueur $/ \mathrm{kg} \mathrm{MS}$ ) notamment parce que GW décrit moins bien le début de la cinétique pour les fourrages, le résidu moyen des 4 premiers points étant 2,4 fois plus élevé qu'avec G3.

En conclusion, le modèle GW fournit une estimation correcte de TSMT mais décrit moins bien le début de la cinétique d'excrétion que les lois gamma. En revanche, ces dernières peuvent être à l'origine de biais importants dans l'estimation du TSMT et il est préférable d'employer une loi gamma de degré 2 pour le transit des concentrés et de degré 3 pour celui, plus lent, des fourrages non broyés. Dans tous les cas, les modèles accroissent la variabilité des estimations de TSMT par rapport aux mesures directes S1 ou S2.

Ellis W. C: Matis J. H., Lascano C., 1979. Fed. Proc., 38, 2702-2706.

Faichney G. J., 1975. Aust. J. agric. Res., 26, 319-327.

Grovum W. L., Williams V. J., 1973. Br. J. Nutr., 30, 313-329.

Peyraud J. L., Mambrini M., Rulquin H., 1988. Reprod. Nutr. Dévelop., 28, 111-112.

Thielemans M. F., François E., Bodart C., Théwis A., 1978. Ann. Biol. anim. Bioch. Biophys., 18, 237-247. 